\title{
Comportamento de Juntas Soldadas de Aço C-Mn Obtidas Pelo Processo GMAW-RE com Eletrodo Rotativo
}

Jeferson Frederico Monteiro Costa ${ }^{1}$, Walker Andrade da Silva Filho ${ }^{1}$, Guilherme Mastrange dos Santos Silveira ${ }^{1}$, Felipe Augusto de Castro Almeida ${ }^{1}$, Marcos Muller Lobato ${ }^{2}$, Tatiane de Campos Chuvas ${ }^{1}$, Matheus Campolina Mendes ${ }^{1}$, Luís Felipe Guimarães de Souza ${ }^{1}$, Jorge Carlos Ferreira Jorge ${ }^{1}$ (1)

${ }^{1}$ Centro Federal de Educação Tecnológica Celso Suckow da Fonseca - CEFET/RJ, Diretoria de Pesquisa e Pós-graduação - DIPPG, Rio de Janeiro, RJ, Brasil.

${ }^{2}$ White Martins Gases Industriais Ltda., Metal Mecânica, Rio de Janeiro, RJ, Brasil.

Como citar: Costa JFM, Silva Filho WA, Silveira GMS, Almeida FAC, Lobato MM, Chuvas TC, et al. Comportamento de juntas soldadas de aço C-Mn obtidas pelo processo GMAW-RE com eletrodo rotativo. Soldagem \& Inspeção. 2019;24:e2411. https://doi.org/10.1590/0104$9224 / \mathrm{SI} 24.11$

Resumo: O presente trabalho tem como objetivo estudar o comportamento de juntas soldadas obtidas pelo processo GMAW com eletrodo rotativo (GMAW-RE), de modo a permitir uma análise comparativa de produtividade e propriedades mecânicas com o processo GMAW convencional (GMAW). Foram realizadas soldagens multipasse pelos dois processos GMAW com preaquecimento de $100{ }^{\circ} \mathrm{C}$ em chapas de dimensões $500 \times 150 \times 10 \mathrm{~mm}$ em aço ASTM A36, na posição plana e energia de soldagem média de $2,30 \mathrm{~kJ} / \mathrm{mm}$. Após a soldagem, foram realizados ensaios de impacto Charpy-V, microdureza e metalográficos em corpos-de-prova removidos transversalmente ao cordão de solda. Adicionalmente, foi realizada uma análise comparativa de produtividade entre os processos. Os resultados mostram que o processo GMAW-RE propicia um aumento de produtividade e redução de custos significativos em relação ao processo GMAW. Adicionalmente, foram observados valores equivalentes de tenacidade ao impacto para o metal de solda, mas uma redução nesta propriedade na zona termicamente afetada. Embora a literatura disponível destaque o elevado potencial deste processo para substituição do processo GMAW convencional em aplicações industriais, verifica-se a necessidade de mais evidências experimentais para um maior suporte a esta indicação.

Palavras-chave: Processo GMAW; Eletrodo rotativo; Produtividade; Propriedades mecânicas.

\section{Behavior of the C-Mn Steel Welded Joints Obtained by GMAW-RE Process with Rotating Electrode}

\begin{abstract}
The main goal of the present work is to evaluate the behavior of welded joints obtained by the GMAW with rotating electrode process (GMAW-RE) in comparison to the Conventional GMAW process (GMAW). Multipass welding by both processes were performed in ASTM A36 steel plates with dimensions of $500 \times 150 \times 10 \mathrm{~mm}$, preheating of $100{ }^{\circ} \mathrm{C}$ in the flat position and average welding energy of $2.30 \mathrm{~kJ} / \mathrm{mm}$. After welding, Charpy-V notch and microhardness tests and metallographic examination were performed in samples removed transversally to the weld bead. Additionally, a comparative analysis of productivity and costs between processes were done. The results show that the GMAW-RE promotes higher productivity and reduction of costs in comparison to GMAW. Additionally, similar impact toughness results were observed in weld metals and a reduction on this property at the HAZ. Although the literature states the high potential of the GMAW-RE for replacement of the conventional GMAW in industrial applications, it was concluded that other experimental results are still necessary before the final conclusion.
\end{abstract}

Key-words: GMAW process; Rotating electrode; Productivity; Mechanical properties.

\section{Introdução}

O aumento das operações de fabricação e reparo de componentes industriais tem motivado o aperfeiçoamento de processos de soldagem, visando a melhoria da produtividade com manutenção de qualidade assegurada e com baixo custo para manter a competitividade.

Neste contexto, é de conhecimento geral que o processo de soldagem GMAW (Gas Metal Arc Welding) é amplamente empregado nas mais diversas aplicações, visto suas características de elevada taxa de deposição, versatilidade, fácil adaptação para automação e custo relativamente baixo em comparação com outros processos a arco elétrico [1-5]. Com o aprimoramento das fontes de potência, o campo de aplicação do processo foi ainda ampliado [6]. 
O desenvolvimento de novos processos com maior produtividade, em geral, é uma forma efetiva de buscar ganhos de competitividade. Entretanto, quando do surgimento de alguma inovação nesta área, faz-se necessária a definição das condições operacionais que efetivamente proporcionam algum benefício ou um melhor desempenho que o processo convencional.

O processo GMAW com eletrodo rotativo (GMAW-RE) é uma variação do processo GMAW, patenteada em 1983 nos EUA, e que foi desenvolvida para a soldagem de uma liga de alumínio usada numa aplicação aeroespacial [7]. A principal característica do processo GMAW-RE é que o arame alimentado continuamente na poça de fusão, como no GMAW, é submetido a um movimento de rotação, com diâmetro e velocidade de rotação pré-estabelecidos [8,9], conforme observado na Figura 1, onde se mostra o exemplo com diâmetro de rotação de $8 \mathrm{~mm}$ e velocidade de rotação de $3000 \mathrm{rpm}$.

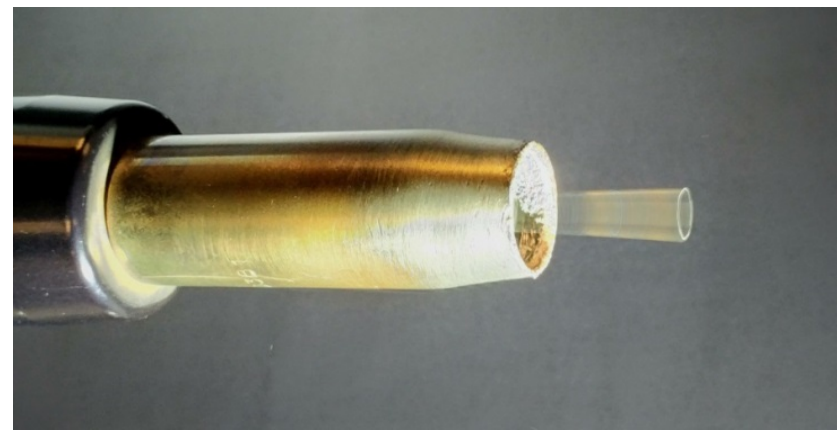

Figura 1. Detalhe da rotação do arame na tocha de soldagem GMAW-RE com diâmetro de giro de $8 \mathrm{~mm}$

O movimento giratório do arame faz com que arco elétrico estabelecido entre este e o metal base, durante a soldagem, seja submetido a esse mesmo movimento de giro. Como o movimento giratório do arame é de pequeno diâmetro, o movimento do arco elétrico contorna a poça de fusão, enquanto a tocha é deslocada ao longo de sua trajetória para formar o cordão de solda. A diferença essencial em relação ao processo GMAW é que enquanto neste processo o arame permanece apontando para o centro da poça de fusão, no GMAW-RE este arame se movimenta continuamente em torno desta.

Uma questão relevante envolve a correta terminologia aplicada ao processo GMAW-RE, visto que vários autores [8,10-13] optaram pela designação do processo como GMAW com arco rotativo. De fato, embora essa designação descreva uma característica do processo, em recente revisão da terminologia dos modos de transferência metálica do processo GMAW, Kah et al. [14] estabeleceram que a transferência metálica rotativa ocorre para faixas de energia mais elevadas, sendo resultado de uma extensão livre de eletrodo longa (25 a $35 \mathrm{~mm}$ ) associado com altas correntes e tensões do arco elétrico. Nesse caso, do processo GMAW convencional, a rotação ocorre apenas no arco elétrico, na ponta do arame, e é provocada pelas forças eletromagnéticas atuantes. Portanto, essa designação para o novo processo como GMAW com arco rotativo pode provocar alguma confusão, caso não seja especificado se a origem da rotação é eletromagnética ou mecânica, em especial, porque se referem a processos com características completamente distintas.

Outros autores [10,15] fazem menção ao processo como GMAW com eletrodo rotativo (GMAW-RE), enquanto que Zhang et al. [16], o designa como processo GMAW com arame rotativo (GMAW-RW). Nesses dois casos a diferença é sutil, pois em geral, o arame utilizado no processo GMAW é também o eletrodo. A exceção ocorre quando há a adição de um arame extra, que pode ser frio ou quente, mas independentemente de ser um ou outro, o arame extra não se configura como um eletrodo no processo. Existem ainda alguns trabalhos $[8,15]$ nos quais é possível encontrar menção ao processo pela sua marca comercial "Spin Arc". Assim como também não é raro encontrar no mesmo texto mais do que uma dessas formas apresentadas, o que demonstra haver uma falta de padronização na designação do processo. No presente trabalho, a designação adotada para o processo é GMAW com eletrodo rotativo (GMAW-RE).

O processo GMAW-RE introduz uma modificação em relação ao convencional que influi no modo de transferência metálica do arame para a poça de fusão e na distribuição de calor na junta $[8,9,12,17,18]$. 0 movimento rotativo acelera as gotículas metálicas radialmente, por efeito da força centrífuga, sendo esse efeito depende do diâmetro e da velocidade de rotação, conforme observado por Guo et al. [13]. Como consequência deste movimento rotativo, são geradas condições mais favoráveis para redução da falta de fusão lateral, que é uma limitação do processo GMAW [19].

Esta característica contribui para viabilizar a soldagem em chanfro estreito, principalmente em juntas de maiores espessuras $[9,20]$, com resultados significativos em relação a redução do volume de metal depositado, tempo de soldagem, preparação da junta e redução nos níveis de tensões residuais.

Segundo Prasad et al. [8], este processo promove um aumento de produtividade e qualidade em soldagens realizadas tanto com arames sólidos quanto tubulares.

Embora alguns estudos [9,13,15,21-24] mostrem resultados sobre as características operacionais do processo, a literatura com evidências sobre as possíveis vantagens competitivas e envolvendo propriedades mecânicas de juntas soldadas 
obtidas por este processo é ainda muito limitada [25-27] e mais evidências experimentais se fazem necessárias para confirmar as afirmações de possíveis melhorias na qualidade e produtividade de soldagens realizadas por este processo [27].

Baseado neste cenário, este trabalho tem por objetivo apresentar resultados sobre o comportamento mecânico e microestrutural de juntas soldadas de aço C-Mn obtidas pelos processos GMAW-RE e GMAW, de modo a contribuir para um melhor entendimento sobre o potencial deste novo processo para aplicações industriais.

\section{Materiais e Métodos}

\subsection{Materiais}

Como material base, foram utilizadas chapas de aço ASTM A36 com microestrutura constituída por ferrita e perlita (Figura 2), com comprimento, largura e espessura nas dimensões de $500 \times 150 \times 10 \mathrm{~mm}$, respectivamente. Como consumível de soldagem, foi utilizado um arame sólido da classe AWS ER 110S-G de 1,2 mm de diâmetro para soldagem por ambos os processos.

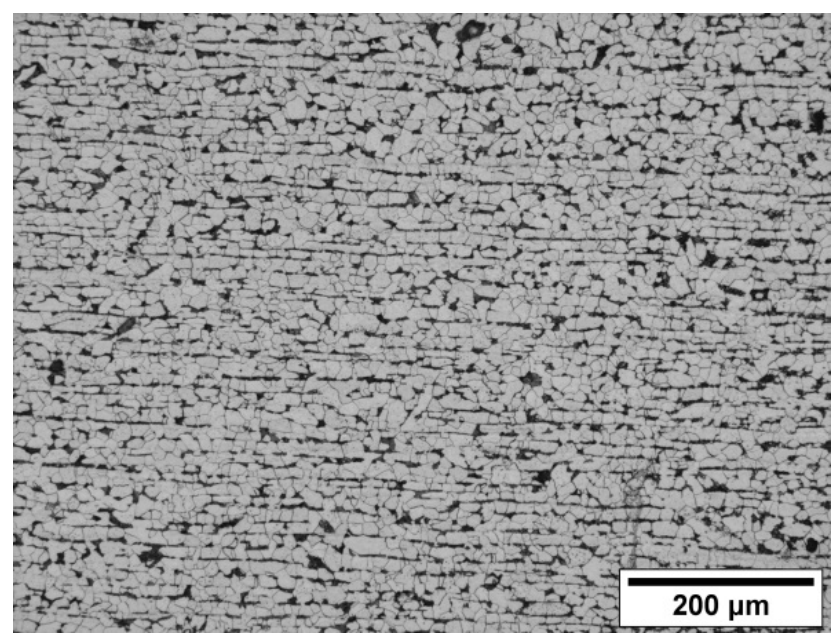

Figura 2. Microestrutura do metal base. Ataque: nital $2 \%$.

A utilização do aço ASTM A36 e de um consumível de alta resistência tem por objetivo avaliar os possíveis efeitos dos diferentes ciclos térmicos nas características de materiais com diferentes temperabilidades em um mesmo estudo.

A Tabela 1 mostra a composição química dos materiais utilizados, obtida por espectroscopia de emissão óptica.

Tabela 1. Composição química dos materiais (\% em peso).

\begin{tabular}{|c|c|c|c|c|c|c|c|c|c|c|}
\hline \multirow{2}{*}{ Material } & \multicolumn{10}{|c|}{ Elemento } \\
\hline & C & $\mathrm{Si}$ & $\mathbf{P}$ & $\mathbf{S}$ & $\mathrm{Mn}$ & Mo & $\mathrm{Ni}$ & $\mathrm{Cr}$ & $\mathrm{Cu}$ & Ceq( $\left(^{*}\right)$ \\
\hline Metal base & 0,22 & 0,14 & 0,017 & 0,020 & 0,71 & 0,022 & 0,093 & 0,033 & 0,02 & 0,44 \\
\hline Metal de solda GMAW & 0,14 & 0,43 & 0,013 & 0,003 & 1,35 & 0,27 & 1,15 & 0,24 & 0,11 & 0,55 \\
\hline Metal de solda GMAW-RE & 0,15 & 0,38 & 0,017 & 0,002 & 1,27 & 0,26 & 1,04 & 0,23 & 0,12 & 0,53 \\
\hline
\end{tabular}

$(*) \mathrm{Ceq}=\mathrm{C}+\mathrm{Mn} / 6+(\mathrm{Cr}+\mathrm{Mo}+\mathrm{V}) / 5+(\mathrm{Cu}+\mathrm{Ni}) / 15$.

\subsection{Soldagem das juntas}

As juntas foram preparadas a partir das chapas com as dimensões apresentadas no item 2.1, consistindo de corte térmico com arco plasma e realização do chanfro por usinagem. Foi utilizada uma fonte de soldagem trifásica inversora SINERGICA CV, com ciclo de trabalho de $450 \mathrm{~A}$ a $100 \%$. A soldagem foi mecanizada através de um sistema composto por um trator acoplado a um trilho magnético, comandados por um controlador. A junta GMAW foi preparada com chanfro $\mathrm{V}-60^{\circ} \mathrm{e}$ com abertura na raiz de 5,0 mm, enquanto a junta GMAW-RE foi preparada com chanfro reto e com abertura de raiz de $8,0 \mathrm{~mm}$, conforme mostrado na Figura 3. 


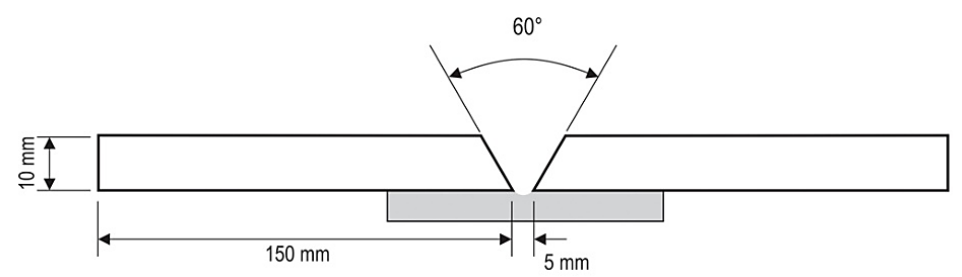

(a)

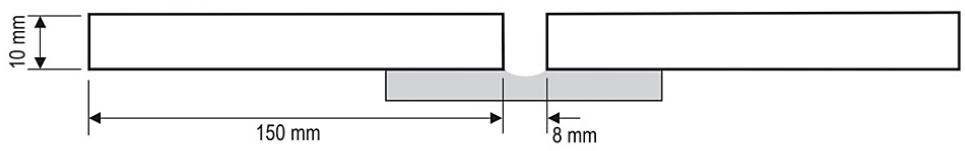

(b)

Figura 3. Detalhes da geometria das juntas utilizadas. (a) GMAW; (b) GMAW-RE.

As juntas foram preaquecidas à temperatura de $100^{\circ} \mathrm{C}$, através de chama oxiacetilênica, e posteriormente foi realizada a soldagem multipasse, na posição plana, com uma temperatura máxima entre passes de $150{ }^{\circ} \mathrm{C}$. $\mathrm{O}$ controle do preaquecimento e da temperatura entre passes foi realizado através de pirômetro de contato, devidamente calibrado. Como gás de proteção, foi utilizada uma mistura $\mathrm{Ar}-2 \% \mathrm{O}_{2}$ com uma vazão de $20 \mathrm{l} / \mathrm{min}$.

A soldagem foi conduzida com oscilação lateral em ambos os processos, de modo a obter energias de soldagem mais próximas e, consequentemente, velocidades de resfriamento similares. O modo de transferência verificado em ambas as soldagens foi o "spray". Foi ainda utilizado um sistema portátil de monitoração de parâmetros de soldagem, que permite registrar a corrente e a tensão do arco elétrico, a vazão do gás de proteção e a velocidade de alimentação de arame-eletrodo, com taxa de aquisição de dados de $5 \mathrm{kHz}$.

Para o processo GMAW-RE, foi utilizada uma velocidade de rotação de $3.000 \mathrm{rpm}$ e um diâmetro de giro de $3 \mathrm{~mm}$.

A Tabela 2 apresenta os parâmetros de soldagem utilizados, obtidos a partir do sistema de monitoração.

Tabela 2. Parâmetros de soldagem das juntas soldadas.

\begin{tabular}{|c|c|c|c|c|c|c|c|c|}
\hline Processo & Passe & I (A) & $U(V)$ & $\begin{array}{c}\text { Vs } \\
(\mathrm{mm} / \mathrm{s})\end{array}$ & $\mathrm{Va}(\mathrm{mm} / \mathrm{s})$ & Es (kJ/mm) & $\begin{array}{c}\text { Tempo de } \\
\text { arco aberto } \\
\text { (s) }\end{array}$ & $\begin{array}{c}\text { Taxa de } \\
\text { deposição } \\
(\mathrm{kg} / \mathrm{h})\end{array}$ \\
\hline \multirow{3}{*}{ GMAW } & 1 & 216 & 26 & 2,2 & 1,0 & 2,5 & 225 & 3,25 \\
\hline & 2 & 251 & 27 & 3,5 & 1,3 & 2,0 & 145 & 4,21 \\
\hline & 3 & 257 & 27 & 3,3 & 1,3 & 2,1 & 153 & 4,22 \\
\hline Média GMAW & & 241 & 27 & 3,0 & - & 2,25 & - & 3,80 \\
\hline \multirow{2}{*}{ GMAW-R } & 1 & 254 & 27 & 3,1 & 1,3 & 2,27 & 163 & 4,22 \\
\hline & 2 & 241 & 27 & 5,5 & 1,3 & 2,39 & 182 & 4,21 \\
\hline Média GMAW-R & & 247 & 27 & 5,8 & - & 2,33 & & 4,21 \\
\hline
\end{tabular}

$\mathrm{I}$ = Corrente; U = Voltagem; Vs = Velocidade de soldagem; Va = Velocidade de alimentação do arame; Es = Energia de soldagem.

O sistema de monitoração de dados de soldagem determina a Energia de Soldagem a partir das Potências Instantâneas $\left(P_{i}\right)$. A descrição matemática é dada pela Equação 1 , onde $V s$ representa a velocidade de soldagem, $l_{i}$ a corrente instantânea, $U_{i}$ a tensão instantânea e $n$ o número das amostras de medição discreta dos sinais elétricos [28].

$$
E s=\frac{P i}{V S}=\sum_{i}^{n} \frac{\left(I_{i} U_{i}\right)}{n} / V s
$$

\subsection{Ensaios mecânicos}

Foram realizados ensaios de impacto Charpy-V à temperatura de $23{ }^{\circ} \mathrm{C}$ em corpos-de-prova reduzidos, nas dimensões de $5 \times 10 \times 55 \mathrm{~mm}$, retirados transversalmente ao cordão de solda e a $2 \mathrm{~mm}$ da superfície da junta, sendo realizados três ensaios para cada condição analisada. $O$ entalhe foi posicionado no plano da espessura nas posições relativas ao centro do cordão de solda, zona termicamente afetada (ZTA) a $1 \mathrm{~mm}$ da linha de fusão (LF) e no metal base. A Figura 4 mostra esquematicamente o posicionamento dos corpos de prova, 


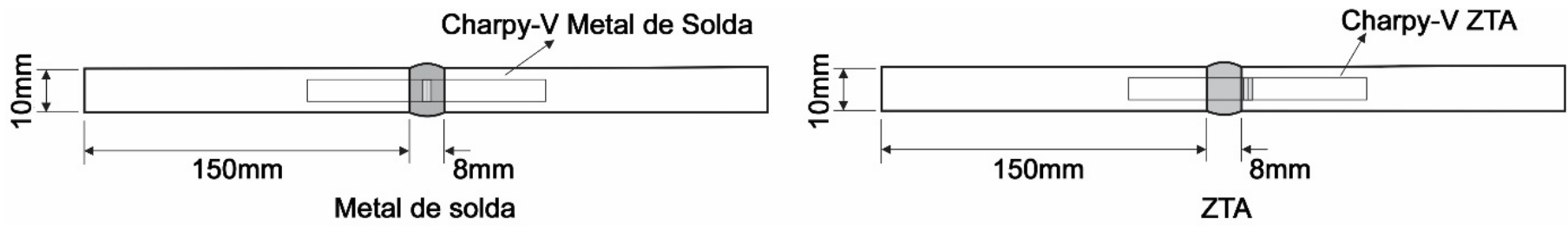

Figura 4. Posição dos corpos de prova para ensaios de impacto Charpy-V na junta obtida com o processo GMAW-RE.

Foram realizados ensaios de microdureza Vickers $\left(\mathrm{HV}_{0.5}\right)$ de acordo com a norma ASTM A 370 [29], com aplicação de carga de 500 gf. em corpos-de-prova transversais ao cordão de solda, sendo realizadas medidas nas posições relativas ao centro dos corpos-de-prova para ensaio de impacto Charpy- $\mathrm{V}$, conforme mostrado na Figura 5.

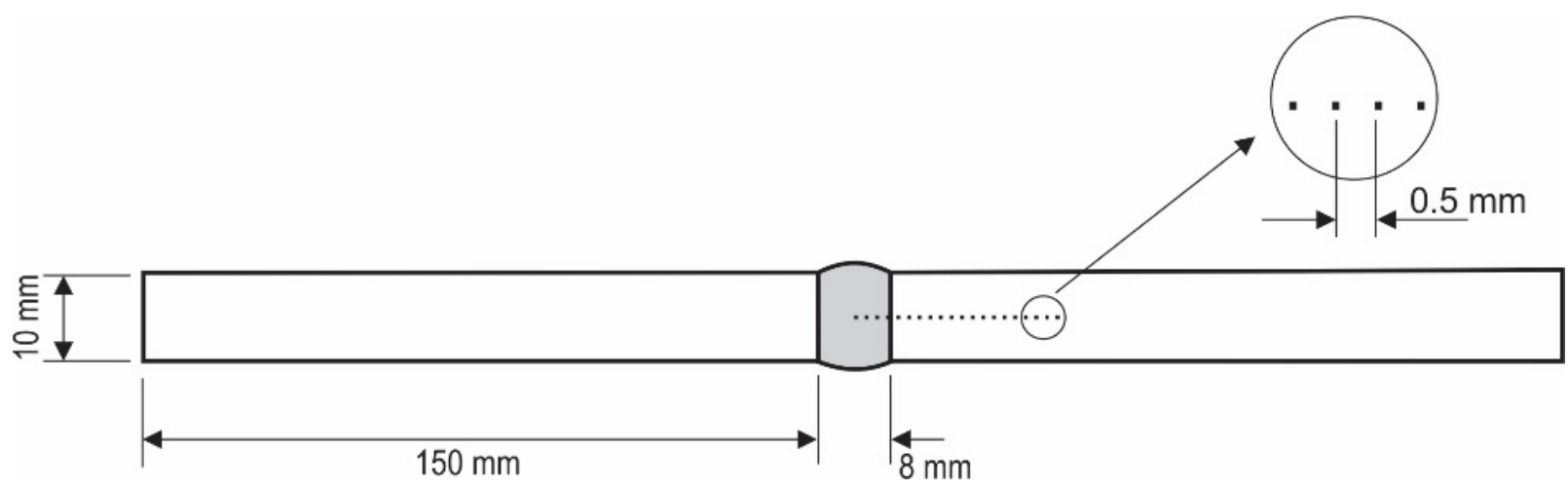

Figura 5. Detalhe da posição dos ensaios de microdureza Vickers realizados na junta obtida com o processo GMAW-RE.

\subsection{Ensaios metalográficos}

Foram realizados ensaios macro e micrográficos por microscopia óptica (MO) e eletrônica de varredura (MEV) para avaliação dos constituintes microestruturais presentes nas diversas regiões das juntas soldadas.

Os ensaios micrográficos foram realizados na região relativa ao entalhe do corpo de prova de impacto Charpy- $V$, de forma a permitir uma análise da relação entre a microestrutura e a tenacidade ao impacto.

No caso da ZTA, onde a variação microestrutural foi mais acentuada ao longo do entalhe Charpy-V, foi realizada uma análise quantitativa da contribuição de cada região com microestrutura diferente, através de observação direta no microscópio ótico, com auxílio de software de análise de imagens para maior precisão nas medições.

O preparo das amostras para análise consistiu do procedimento convencional de lixamento e polimento com pasta de diamante com granulometrias $6,3,1$ e $1 / 4 \mu \mathrm{m}$, seguido de ataque químico com o reagente nital $2 \%$.

\section{Resultados}

\subsection{Ensaios metalográficos}

A Figura 6 mostra o aspecto dos cordões de solda obtidos por ambos os processos, onde se nota um comportamento semelhante, sem ocorrência de defeitos de soldagem e com uma menor incidência de respingos para o processo GMAW-RE. 


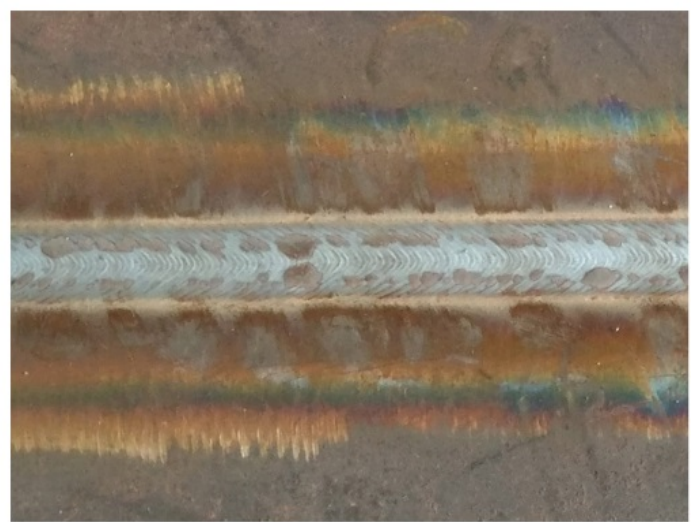

GMAW (superfície)

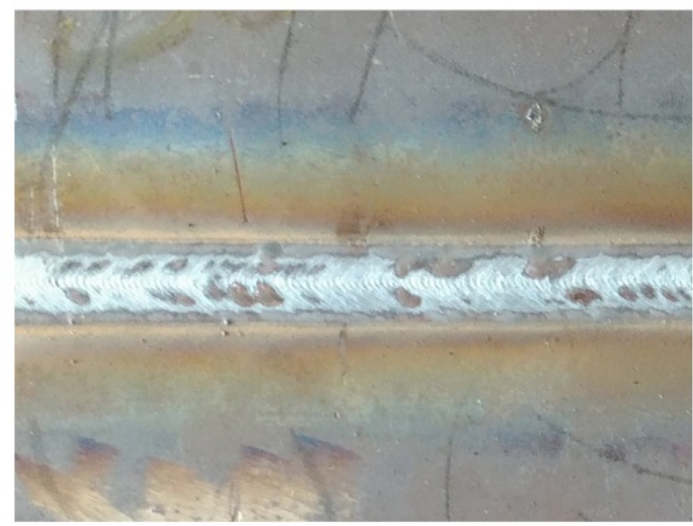

GMAW-RE (superfície)
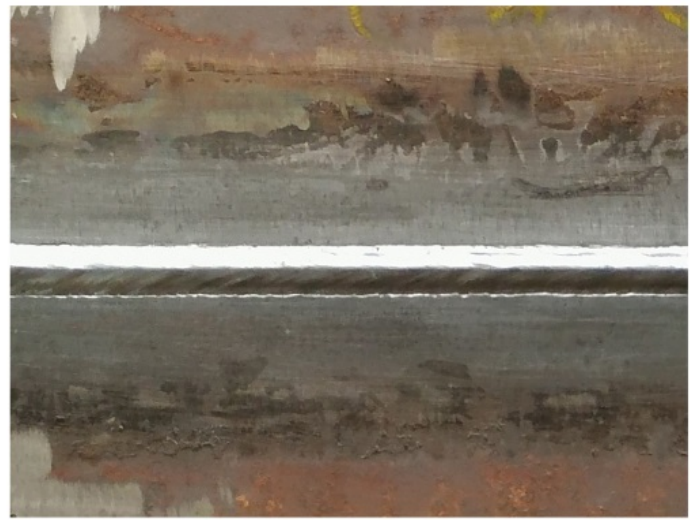

GMAW (raiz)
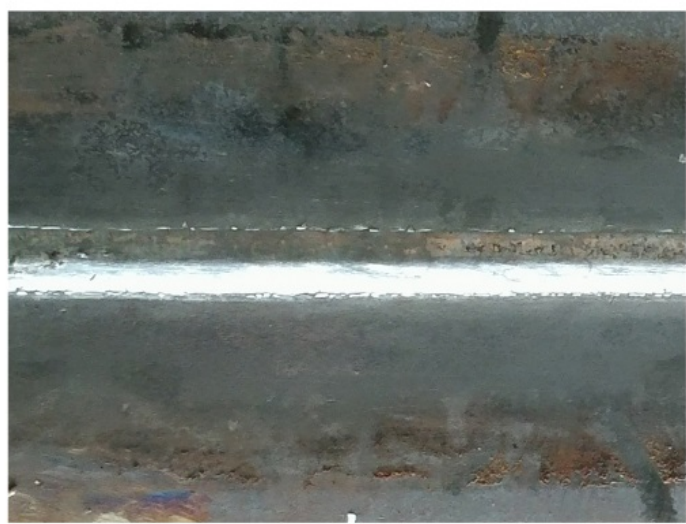

GMAW-RE (raiz)

Figura 6. Aspecto dos cordões de solda das juntas.

A Figura 7 mostra a macrografia das juntas soldadas, onde se nota o aspecto da soldagem multipasse. A inspeção visual da seção macrográfica não revela a presença de descontinuidades. Nota-se ainda uma maior extensão da ZTA para o processo GMAW-RE.

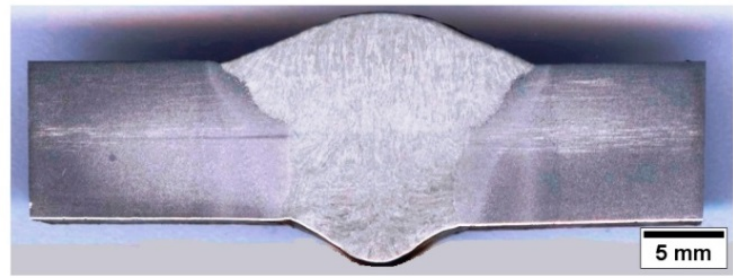

GMAW

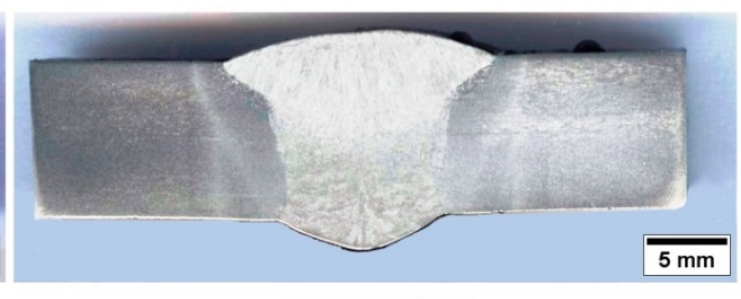

GMAW-RE

Figura 7. Macrografia das juntas soldadas. Ataque: nital $2 \%$.

A Figura 8 apresenta a evolução microstrutural ao longo da ponta do entalhe Charpy-V posicionado na ZTA das juntas soldadas, onde se percebe a contribuição de diferentes constituintes microestruturais. Um melhor detalhamento destes constituintes e sua contribuição percentual é apresentado na Figura 9, com destaque para o fato que a junta soldada pelo processo GMAW-RE apresenta uma microestrutura muito mais grosseira. 


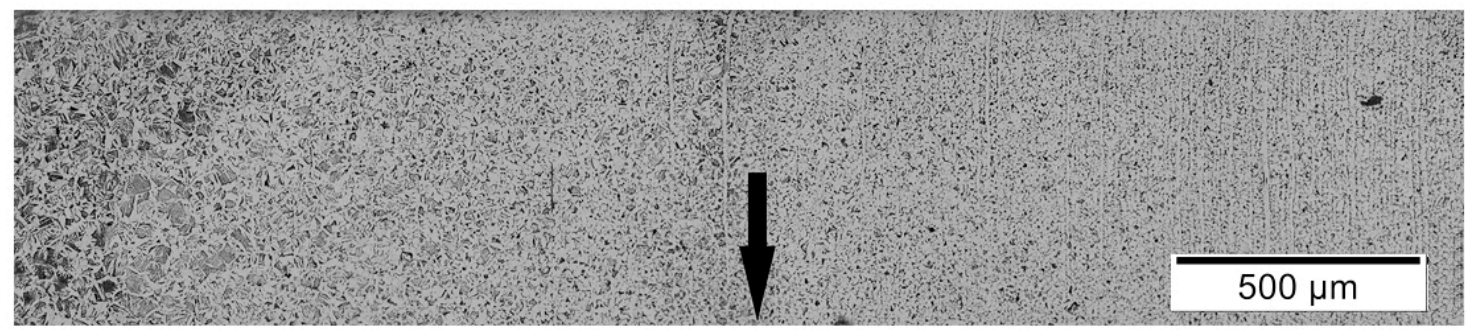

(a)

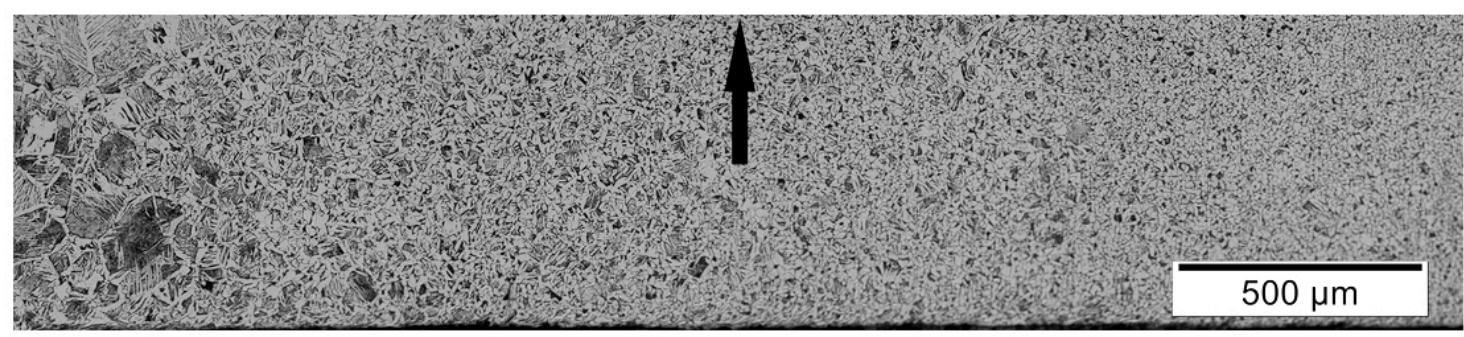

(b)

Figura 8. Distribuição microestrutural na ponta do entalhe Charpy-V (indicada pela seta) posicionado na ZTA das juntas soldadas. Ataque: nital 2\%. (a) GMAW; (b) GMAW-RE.

Já no metal de solda, se nota uma maior homogeneidade microestrutural. Embora se verifique a presença de constituintes usualmente observados em metais de solda de aço de alta resistência da classe $760 \mathrm{MPa}$ (110 ksi), observa-se uma microestrutura ligeiramente mais refinada e com menor incidência de constituintes A-M para o processo GMAW-RE (Figura 10).

\subsection{Ensaios mecânicos}

A Figura 11 mostra os resultados dos ensaios de impacto realizados nas diversas condições de análise, onde se nota que o processo GMAW-RE apresentou em comparação ao processo GMAW, resultados similares para o metal de solda e uma redução significativa na ZTA. Nota-se que somente a ZTA da junta obtida com o processo GMAW-RE apresentou resultado de tenacidade ao impacto inferior ao metal base.

Os resultados dos ensaios de microdureza Vickers revelaram comportamento similar para as juntas soldadas, acompanhando as microestruturas obtidas (Figura 12).

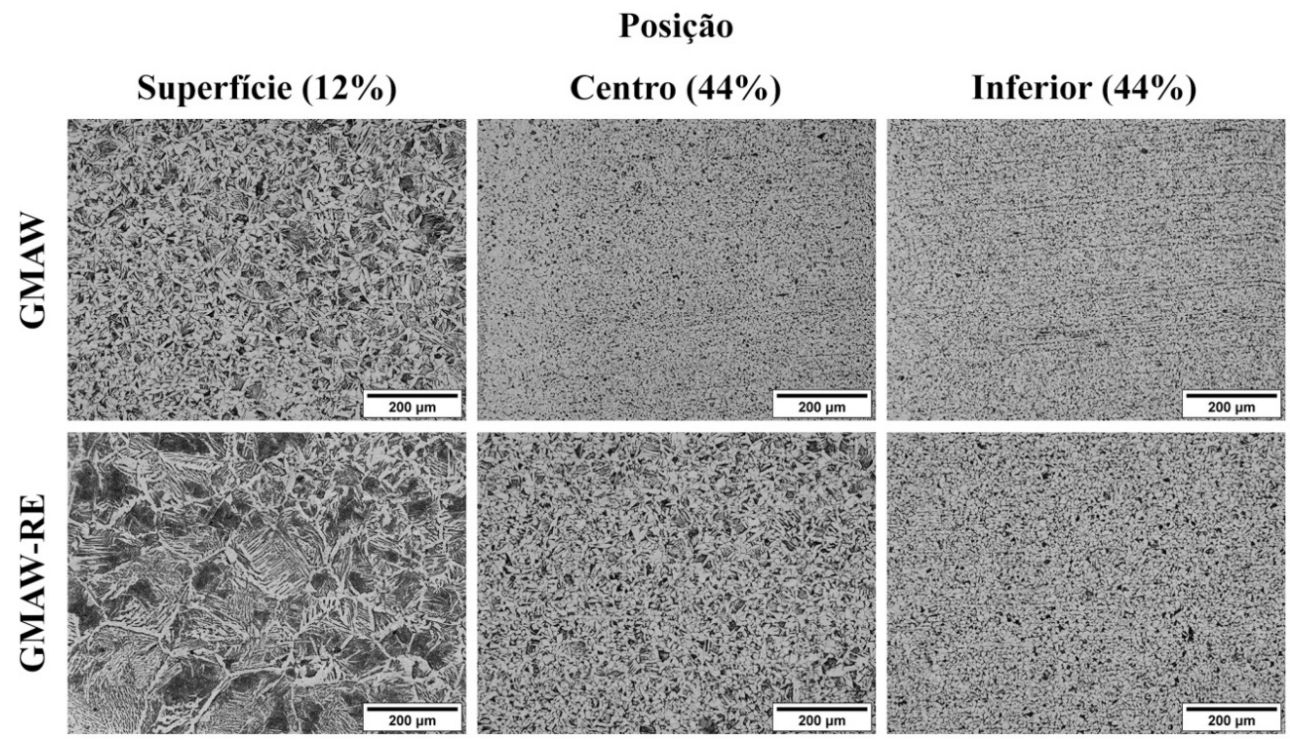

Figura 9. Microestrutura da ZTA na região correspondente à ponta do entalhe do corpo-de-prova Charpy-V. Onde: Os percentuais apresentados na legenda superior, se referem a participação de cada região no entalhe Charpy-V. Ataque: nital2\%. 

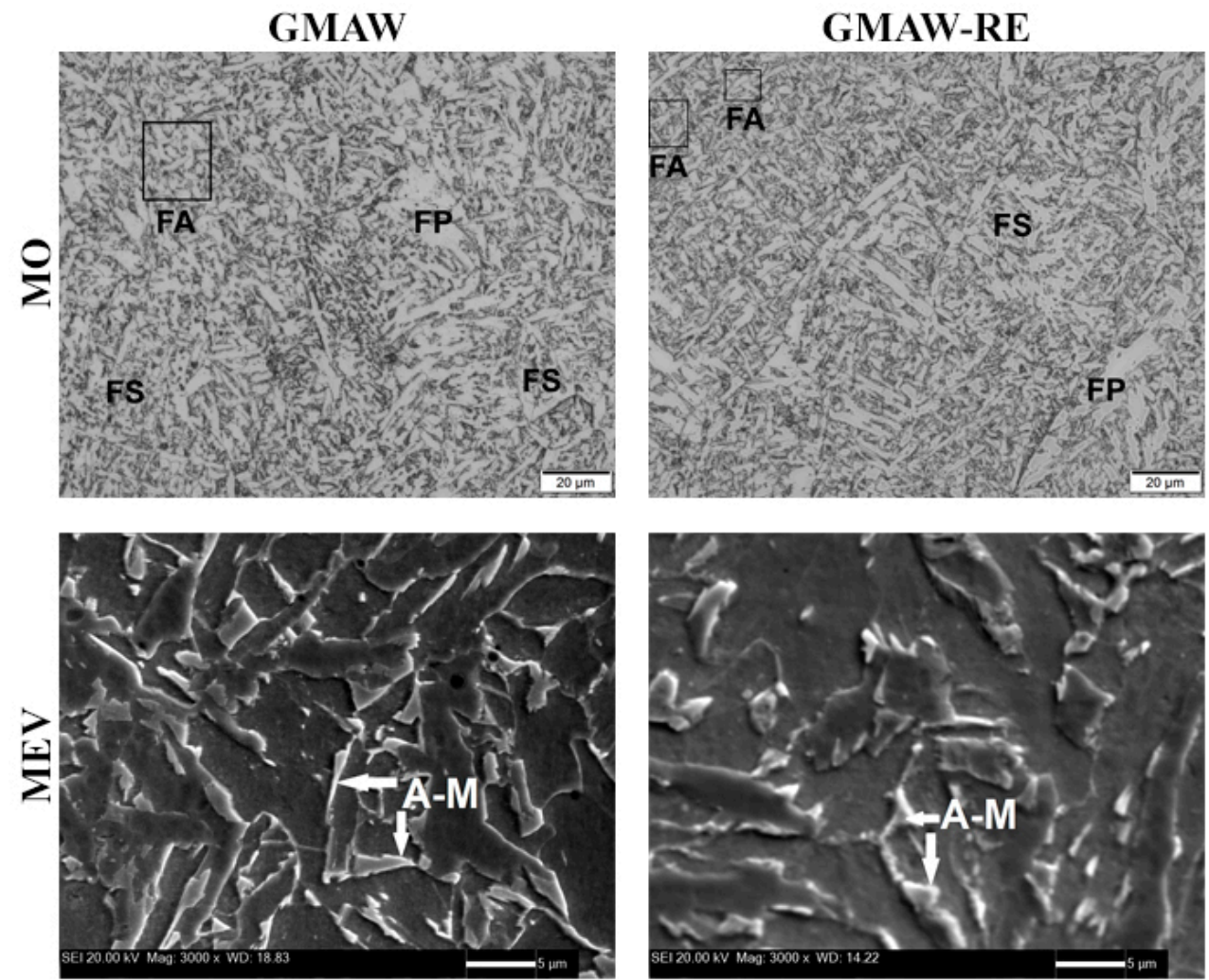

Figura 10. Microestrutura do metal de solda na região correspondente ao entalhe do corpo-de-prova Charpy-V. Ataque: nital $2 \%$. Onde: FA = Ferrita Acicular; FS = Ferrita com Segunda Fase; FP = Ferrita Primária; A-M = Constituinte A-M.

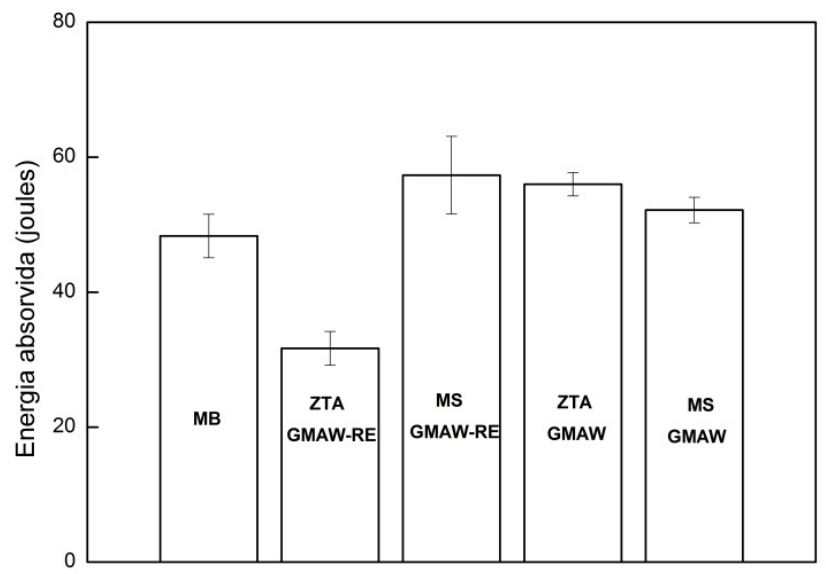

Figura 11. Resultados dos ensaios de impacto Charpy-V realizados a $23^{\circ} \mathrm{C}$, em joules. Onde: $\mathrm{MB}=\mathrm{Metal}$ base; $\mathrm{ZTA}=\mathrm{Zona}$ termicamente afetada; MS = Metal de solda. 


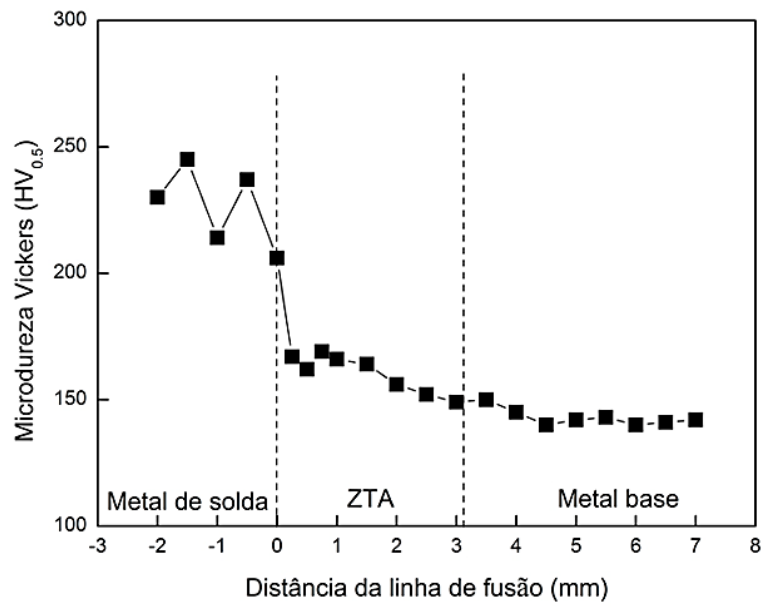

(a)

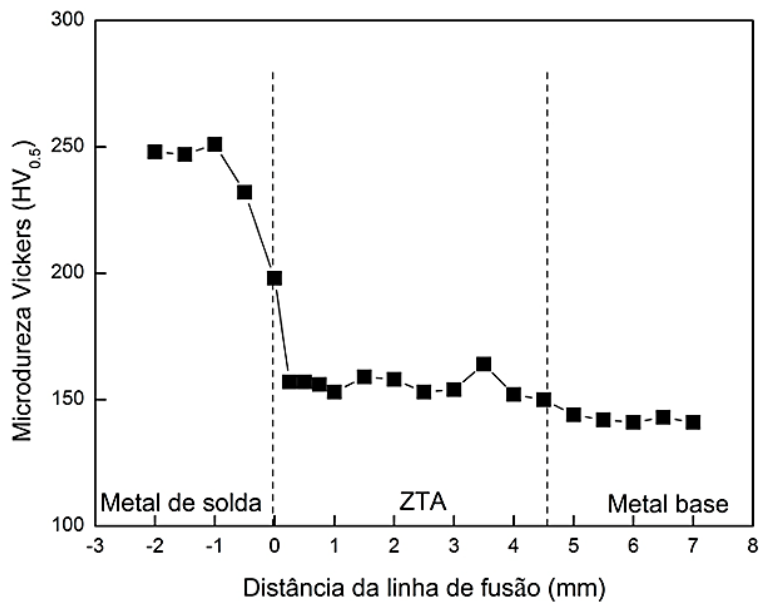

(b)

Figura 12. Resultados dos ensaios de microdureza Vickers $\left(\mathrm{HV}_{0.5}\right)$ realizados na região central do corpo-de-prova de impacto Charpy-V. (a) GMAW; (b) GMAW-RE.

\section{Discussão}

\subsection{Produtividade}

Segundo Myers [30] a taxa de deposição para o processo GMAW utilizando um arame sólido de 1,2 mm de diâmetro varia de 3,6 a 4,5 kg/h. Como observado na Tabela 3, os valores obtidos no presente trabalho situam-se dentro destes limites para ambos os processos, estando de acordo com outros trabalhos experimentais [30-35]. Embora a taxa de deposição média observada com o processo GMAW seja inferior ao do processo GMAW-RE, deve-se destacar que este resultado é consequência da contribuição do passe de raiz, visto que os passes de enchimento e acabamento apresentaram resultados similares (Tabela 2). Neste aspecto, deve-se comentar que os parâmetros de soldagem para o passe de raiz do processo GMAW foram ajustados experimentalmente para obtenção do passe de raíz com a qualidade desejada. Para encontrar os parâmetros do GMAW-RE, foi realizada uma série de testes preliminares, variando o diâmetro de rotação, velocidade de rotação e abertura da junta [36], onde os parâmetros utilizados neste artigo foram os que representam as melhores condições de soldagem, inclusive reprodutibilidade.

Outra questão importante envolve o fato que, mesmo utilizando uma espessura que se encontra no limite indicado por outros autores [23] para a qual existe ganho na aplicação do processo GMAW-RE, os resultados mostrados na Tabela 3 permitem verificar que o desempenho deste processo é ainda significativamente superior aquele propiciado pelo processo convencional.

Conforme já citado, a obtenção de juntas soldadas sem ocorrência de falta de fusão lateral mesmo com utilização de chanfro reto, a grande limitação do processo convencional [19], traz como consequência uma redução importante do tempo de arco aberto, menor volume de metal depositado, menor consumo de gás para soldagem, menor tempo para preaquecimento, menor necessidade de preparação de chanfro, entre outros. A combinação destes resultados permite um aumento da produtividade associada com a redução dos custos de soldagem da ordem de $30 \%$ no caso do presente estudo.

Tabela 3. Desempenho comparativo entre os processos GMAW e GMAW-RE por metro de solda.

\begin{tabular}{cccc}
\hline Item & GMAW & GMAW-RE & Variação (\%) \\
\hline Metal depositado $(\mathrm{kg})$ & 1,10 & 0,81 & 27 \\
Tempo de arco aberto $(\mathrm{min})$ & 17,4 & 11,5 & 34 \\
Volume de Gás (L) & 349 & 230 & 34 \\
Taxa de deposição média (kg/h) & 3,80 & 4,21 & 11 \\
\hline
\end{tabular}

Para obter o retorno do investimento do equipamento, seria necessária a fabricação de 4285 metros de junta. Entretanto, deve-se ressaltar que uma das principais vantagens do processo é o ganho de produtividade. Para soldar essa quantidade de metros de juntas seriam necessárias 820 horas de operação com o novo processo (GMAW-RE), contra 1245 horas para o GMAW convencional, uma redução de 425 horas de trabalho.

Estes cálculos foram baseados nos dados das juntas deste trabalho, em que os custos de fabricação do chanfro não foram considerados, fator que tornaria o retorno mais rápido. 


\subsection{Propriedades da junta soldada}

Como comentado previamente, o desenvolvimento do processo GMAW-RE tem como um de seus objetivos principais a obtenção de junta soldada de boa qualidade com utilização de chanfro reto ao invés de junta chanfrada usualmente empregada pelo processo convencional. Assim, diferentes geometrias de junta foram intencionalmente utilizadas para simular as condições reais de emprego de cada processo. Estas alterações na geometria da junta (Figura 3), fizeram com que as condições de soldagem para cada processo fossem diferentes de modo a obter juntas isentas de defeitos para análise das propriedades mecânicas. No caso da junta GMAW-RE foi utilizado chanfro reto com abertura de raiz maior do que a aplicada na junta GMAW, enquanto neste último processo, foi necessário um ajuste dos parâmetros de soldagem para obtenção de passe de raiz adequado, além do chanfro em $\mathrm{V}-60^{\circ}$.

Estas alterações, notadamente na geometria de chanfro (Figura 3), poderiam indicar também uma redução da largura da poça de fusão e da extensão da ZTA para a junta GMAW-RE, o que foi observado por outros autores [20,26], quando em soldagem em espessuras maiores. No entanto, no caso do presente trabalho, onde a espessura de $10 \mathrm{~mm}$ se situa no limite para a qual o processo GMAW-RE seria vantajoso [23], este fato não foi observado visto que a extensão da ZTA é muito superior no processo GMAW-RE (da ordem de 3,1 $\mathrm{mm}$ para o processo GMAW e 4,6 mm para o processo GMAW-RE) enquanto que a largura da poça da fusão é apenas ligeiramente inferior (da ordem de 11,1 mm para o processo GMAW e 10,4 mm para o processo GMAW-RE na região central da junta).

Uma análise mais detalhada das macrografias apresentadas na Figura 5 permite observar uma extensão da ZTA maior para o processo GMAW-RE, o que não é devido somente a maior energia de soldagem (Tabela 2). De fato, embora a energia de soldagem deste processo seja ligeiramente superior, há de se ressaltar que o grande diferencial destes 2 processos envolve exatamente o fato do eletrodo ser rotativo. No processo convencional a energia do arco é concentrada no centro do cordão, enquanto que no GMAW-RE, a rotação do arame faz com que a energia seja direcionada de forma periódica para as paredes do chanfro, modificando a distribuição de calor na junta [22,23,26,37]. Assim, segundo Bai et al. [24], a extensão da largura da ZTA no processo GMAW-RE tende a ser maior devido a rotação do arco, o que resulta em uma diminuição na energia no centro do cordão de solda e um aumento na região periférica, conforme observado por Guo et al. [37].

Outro efeito que pode ser esperado em função da rotação do arco elétrico é o aumento da largura da poça de fusão, que por sua vez, terá efeito sobre a densidade potência do processo, ou seja, o aumento da largura da poça de fusão diminui a densidade de potência e a penetração na junta. Espera-se ainda que, com o calor redistribuído para as paredes do chanfro pela rotação do arco, o fluxo térmico na direção transversal ao deslocamento da fonte de calor seja mais intenso, o que contribui para uma maior extensão da ZTA. Como consequência, a microestrutura da ZTA deverá mais grosseira, por experimentar um ciclo térmico com maiores temperaturas de pico e permanência por tempos mais longos em temperaturas mais elevadas, o que foi observado no presente trabalho (Figura 7).

No caso presente, como se trata de um aço C-Mn de baixa temperabilidade, os constituintes microestruturais presentes em ambas as juntas são os esperados para um aço C-Mn, somente diferindo no tamanho de grão em função do ciclo térmico experimentado por cada um. $O$ efeito do reaquecimento pelos passes posteriores se traduz em um refino microestrutural e ocorrência preponderante de ferrita e carbetos na região central (Figura 7), todas estas evidências estando de acordo com outros trabalhos experimentais [38-43].

Já no metal de solda, também como esperado [16], o comportamento foi inverso com o processo GMAW-RE o qual resultou em uma microestrutura mais refinada e apresentando os mesmos constituintes microestruturais (Figura 8 ), os quais são os característicos para um metal de solda da classe $760 \mathrm{MPa}(110 \mathrm{ksi})[31,44,45]$.

A similaridade microestrutural para o metal de solda de ambas as juntas, foi observada no comportamento mecânico da junta soldada, pois como pode ser notado nas Figuras 9 e 10, os valores de microdureza e de tenacidade ao impacto são próximos para as duas juntas soldadas.

O mesmo efeito também foi notado na ZTA, embora com comportamento diferente. Neste caso, uma contribuição superior de microestrutura mais grosseira na ponta do entalhe Charpy- $\mathrm{V}$, contribuiu para uma redução significativa na tenacidade ao impacto da junta soldada pelo processo GMAW-RE. No entanto, deve ser destacado o fato que na soldagem de aços como o aço ASTM A36 utilizado no presente trabalho, não existem requisitos de tenacidade ao impacto para o metal base [46]. Assim, a qualificação de procedimentos que, usualmente apresenta requisitos envolvendo ensaios de tração e dobramento, pode ser realizada com resultados satisfatórios, conforme verificado recentemente por Silveira [36].

Estes resultados estão de acordo com a literatura $[47,48]$, que comenta ser esta uma questão importante para discussão. De fato, o posicionamento do entalhe Charpy-V a uma mesma distância da linha de fusão para condições diferentes de distribuição de calor na junta soldada, pode provocar efeitos importantes nas propriedades mecânicas, uma vez que estruturas mais grosseiras submetidas a ciclos de resfriamento mais longos podem apresentar resultados de tenacidade inferiores.

\subsection{Aspectos práticos dos resultados}

Os resultados obtidos no presente trabalho objetivaram contribuir para um melhor entendimento do desempenho do processo GMAW-RE na proposta de substituição do processo GMAW em aplicações industriais. Neste aspecto, foi confirmado o elevado potencial para prover as vantagens competitivas de ganho substancial de produtividade e redução de custos, 
mesmo para chapas mais finas. Isto permite inferir que em juntas mais espessas, os resultados neste aspecto podem ser ainda mais representativos.

No entanto, deve ser destacado que uma análise mais decisiva sobre as reais vantagens da utilização deste processo, ainda precisa de mais evidências que consolidem a tendência observada. Particularmente, algumas questões merecem um destaque para futuras avaliações, tais como, possíveis vantagens de utilização de aquisição de um equipamento (tocha de soldagem) de custo elevado para um retorno financeiro adequado, possibilidade de soldagem fora de posição e sensibilidade a preparação da superfície da junta soldada. Uma análise inicial permitiu verificar que, sob o ponto de vista de investimento, a realização de soldagem a partir de 4.500 metros de juntas realizadas, já traria uma resposta positiva neste quesito, visto não ser apenas o custo do equipamento o fator fundamental. A redução do tempo de preparação de chanfro e do tempo de soldagem e suas consequências, também são fatores de alto impacto.

Em relação às propriedades mecânicas das juntas soldadas, fica evidente a necessidade de realização de outros experimentos que avaliem o comportamento de diversos outros materiais, espessuras e mesmo modificação de parâmetros de soldagem, visto que para procedimentos similares parece haver um compromisso das propriedades da ZTA em função do ciclo térmico com maiores temperaturas de pico. Neste aspecto, deve-se ainda considerar que na soldagem de aços de alta temperabilidade, esta questão pode até contribuir para um menor risco de ocorrência de trincas a frio, podendo ser este um fator favorável.

De uma maneira geral, parece haver uma indicação para o estudo prioritário da ZTA, região que é submetida aos maiores efeitos negativos da movimentação do arco, visto que no metal de solda estes efeitos parecem não provocar mudanças significativas ou mesmo apresentar vantagens devido ao refino microestrutural, conforme observado no presente trabalho, onde a utilização de metal de solda de aço de alta resistência mecânica deve ser ainda destacada.

\section{Conclusões}

Do exposto no transcurso do presente trabalho, pode-se concluir para as condições experimentais utilizadas que:

a) O processo GMAW-RE propiciou a obtenção de juntas soldadas sem ocorrência de falta de fusão lateral mesmo com utilização de chanfro reto, com reduções significativas do tempo de arco aberto, volume de metal depositado, consumo de gás de proteção e menor necessidade de preparação de chanfro;

b) O processo GMAW com eletrodo rotativo (GMAW-RE) permitiu um ganho de produtividade e redução de custos da ordem de $30 \%$ em relação ao processo GMAW convencional;

c) O processo GMAW-RE possibilitou a soldagem de juntas com propriedades mecânicas similares às obtidas com o processo GMAW no metal de solda. Na ZTA os efeitos do ciclo térmico diferenciado provocaram uma redução da tenacidade ao impacto devido às mudanças microestruturais observadas;

d) Embora o processo GMAW-RE apresente um elevado potencial para substituição do processo GMAW em diversas aplicações industriais, são necessárias mais evidências experimentais sobre o comportamento mecânico e microestrutural de juntas soldadas produzidas por este processo antes de uma conclusão definitiva sobre este assunto.

\section{Agradecimentos}

Os autores agradecem às Instituições pelo apoio prestado na execução do presente trabalho: CEFET/RJ, White Martins Gases Industriais Ltda., Centro Tecnológico do Exército (CTEx), CAPES, CNPq e FINEP.

\section{Referências}

[1] Chauhan J. Review paper on gas metal arc welding (GMAW) of mild steel 1018 by using Taguchi technique. International Journal of Current Engineering and Scientific Research. 2017;4(7):57-62.

[2] Paula MPM, Modenesi PJ, Trindade VB. Análise da influência de parâmetros de soldagem em características microestruturais e mecânicas de juntas soldadas de um tubo de aço API X70Q para aplicação Sour Service. Soldagem e Inspeção. 2018;23(2):180-190. http://dx.doi.org/10.1590/0104-9224/si2302.06.

[3] Saha MK, Das S. Gas metal arc weld cladding and its anti-corrosive performance: a brief review. Athens Journal of Technology and Engineering. 2018;5(2):155-174.

[4] Jorge JCF, Meira OG, Madalena FCA, Souza LFG, Araujo LS, Mendes MC. Evaluation of the AISI 904L alloy weld overlays obtained by GMAW and electro-slag welding processes. Journal of Materials Engineering and Performance. 2017;26(5):2204-2212. http://dx.doi.org/10.1007/s11665-017-2631-9.

[5] Devakumaran K, Ananthapadmanaban MR, Ghosh PK. Variation of chemical composition of high strength low alloy steels with different groove sizes in multi-pass conventional and pulsed current gas metal arc weld depositions. Defence Technology. 2015;11(2):147-156. http://dx.doi.org/10.1016/j.dt.2014.11.001. 
[6] Norrish J. Recent Gas Metal Arc Welding (GMAW) process developments: the implications related to international fabrication standards. Welding in the World. 2017;61(4):755-767. http://dx.doi.org/10.1007/s40194-017-0463-8.

[7] Roen RA. Consumable arc welding torch. United States patent US 4,401,878. 1983 Aug 30.

[8] Prasad V, Babu M, Ajay P. A Review on rotating arc welding process. Materials Today: Proceedings. 2018;5:3551-3555.

[9] Wang JY, Ren YS, Yang F, Guo HE. Novel rotation arc system for narrow gap MAG welding. Science and Technology of Welding and Joining. 2007;12(6):505-507. http://dx.doi.org/10.1179/174329307X213756.

[10] Raju D, Raju G. Effect of using a rotating electrode in GMAW on weld bead characteristic of aluminum alloy 6061-T6 weldment. In: Proceedings of the World Congress on Engineering 2016; 2016; London, U.K. Vol. 2. London: International Association of Engineers; 2016.

[11] Duan B, Wang J, Lu Z, Zhang G, Zhang C. Parameter analysis and optimization of the rotating Arc NG-GMAW welding process. International Journal of Simulation Modelling. 2018;17(1):170-179. http://dx.doi.org/10.2507/IJSIMM17(1)CO4.

[12] Li W, Gao K, Wu W, Wang J, Ji Y. Groove sidewall penetration modeling for rotating narrow gap MAG welding. International Journal of Advanced Manufacturing Technology. 2015;78(1-4):573-581. http://dx.doi.org/10.1007/s00170-014-6678-6.

[13] Guo N, Han Y, Jia C, Du Y. Effects of wire rotating frequency on metal transfer process in rotating arc narrow gap horizontal GMAW. Advanced Materials Research. 2011;189-193:3395-3399. http://dx.doi.org/10.4028/www.scientific.net/AMR.189-193.3395.

[14] Kah P, Latifi H, Suoranta R, Martikainen J, Pirinen M. Usability of arc types in industrial welding. International Journal of Mechanical and Materials Engineering. 2014;9(15):1-12. http://dx.doi.org/10.1186/s40712-014-0015-6.

[15] Santos A.G.M., Brito H.O., Direne H.F., Okuyama M.P., Schwedersky M.B., Silva R.H.G. Soldagem MIG/MAG com eletrodo rotativo destinado a tubulações. In: Anais da Rio Oil \& Gas Expo and Conference; 2018; Rio de Janeiro. Rio de Janeiro: Rio Oil \& Gas; 2018. p. 1 10.

[16] Zhang $\mathrm{H}$, Chang $\mathrm{Q}$, Liu J, Lu H, Wu H, Feng J. A novel rotating wire GMAW process to change fusion zone shape and microstructure of mild steel. Materials Letters. 2014;123:101-103. http://dx.doi.org/10.1016/j.matlet.2014.03.018.

[17] Guo N, Lin SB, Gao C, Fan CL, Yang CL. Study on elimination of interface defects in horizontal joints made by rotating arc narrow gap welding. Science and Technology of Welding and Joining. 2009;14(6):584-586. http://dx.doi.org/10.1179/136217109X456942.

[18] Guo N, Wang M, Guo W, Yu J, Feng J. Study on forming mechanism of appearance defect in rotating arc narrow gap horizontal GMAW. International Journal of Advanced Manufacturing Technology. 2014;75(1-4):15-20. http://dx.doi.org/10.1007/s00170-014-6127-6.

[19] Guzman-Flores I, Vargas-Arista B, Gasca-Dominguez J, Cruz-Gonzales CE, González-Albarrán MA, Prado-Villasana J. Effect of torch weaving on the microstructure, tensile and impact resistances, and fracture of HAZ and weld bead by robotic GMAW process on ASTM A36 steel. Soldagem e Inspeção. 2017;22(1):72-86. http://dx.doi.org/10.1590/0104-9224/si2201.08.

[20] Min O, Xin-hua T, Feng-gui L, Shun Y. Welding of quenched and tempered steels with high-spin arc narrow gap MAG system. International Journal of Advanced Manufacturing Technology. 2011;55(5-8):527-533. http://dx.doi.org/10.1007/s00170-010-3052-1.

[21] Yang CL, Guo N, Lin SB, Fan CL, Zhang YQ. Application of rotating arc system to horizontal narrow gap welding. Science and Technology of Welding and Joining. 2009;14(2):172-177. http://dx.doi.org/10.1179/136217108X388651.

[22] Xu WH, Dong CL, Zhang YP, Yi YY. Characteristics and mechanisms of weld formation during oscillating arc narrow gap vertical up GMA Welding. Welding in the World. 2017;61(2):241-248. http://dx.doi.org/10.1007/s40194-017-0425-1.

[23] Xu G, Li L, Wang J, Zhu J, Li P. Study of weld formation in swing arc narrow gap vertical GMA welding by numerical modeling and experiment. International Journal of Advanced Manufacturing Technology. 2018;96(5-8):1905-1917. http://dx.doi.org/10.1007/s00170018-1729-z.

[24] Bai Q, Guo N, Han Y, Zhang J, Wang M. Analysis of temperature distribution in rotating arc welding. Advanced Materials Research. 2012;472-475:1346-1352. http://dx.doi.org/10.4028/www.scientific.net/AMR.472-475.1346.

[25] Shinji I, Masatoshi M, Yuji K. Application of narrow gap welding process with high speed rotating arc to box column joints of heavy thick plates. JFE Technical Report. 2009;14(12):16-21.

[26] Xu WH, Lin SB, Fan CL, Yang CL. Evaluation on microstructure and mechanical properties of high-strength low-alloy steel joints with oscillating arc narrow gap GMA welding. International Journal of Advanced Manufacturing Technology. 2014;75(9-12):1439-1446. http://dx.doi.org/10.1007/s00170-014-6225-5.

[27] Venkatesh G, Sundararaj P, Verma DK, Arulkumar J. Comparison of mechanical and metallurgical properties of conventional GMAW with spin arc GMAW process for carbon steel SA 515. International Research Journal of Engineering and Technology. 2018;5(5):2716-2721.

[28] Scotti A, Reis RP, Liskevych O. Modelo descritivo do fluxo de calor em soldagem a arco visando o conceito de calor imposto efetivo. Soldagem e Inspeção. 2012;17(2):166-172. http://dx.doi.org/10.1590/S0104-92242012000200010.

[29] American Society for Testing and Materials. ASTM A-370-05: standard test methods and definitions for mechanical testing of steel products. West Conshohocken: ASTM; 2005. $47 \mathrm{p}$. 
[30] Myers D. Metal cored wires: advantages and disadvantages. Welding Journal. 2002;81(9):39-42.

[31] Lins AS Jr, Costa HRM, Souza LFG, Jorge JCF. Propriedades mecânicas e microestruturais de juntas soldadas do aço HY-80 pelos processos eletrodo revestido e GMAW. Soldagem e Inspeção. 2014;19(3):200-211. http://dx.doi.org/10.1590/0104-9224/SI1903.02.

[32] Rutzinger R. Influence of the welding process to the dilution rate of weld overlays on unalloyed steel using the weld consumable ERNiCrMo-3 (Alloy 625). BiuletynInstytutu Spawalnictwa. 2014;5:72-75.

[33] Gomes AJC, Queiroz SS, Jorge JCF, Souza LFG, Mendes MC, Araújo LS. Propriedades mecânicas e microestrutura de metais de solda de alta resistência obtidos pelos processos GMAW e SMAW. In: Anais do 72ํ Congresso Anual da ABM; 2017; São Paulo. São Paulo: ABM; 2017.

[34] Soeiro JC Jr, Luz MA, Brandi SD. Comparação da taxa e eficiência de deposição entre os consumíveis ER70S-6 e E71T-1C. Soldagem e Inspeção. 2015;20(1):2-15. http://dx.doi.org/10.1590/0104-9224/SI2001.02.

[35] Jorge JCF, Monteiro JLD, Gomes AJC, Bott IS, Souza LFG, Mendes MC, et al. Influence of welding procedure and PWHT on HSLA steel weld metals. Journal of Materials Research and Technology. 2019;8(1):561-571. http://dx.doi.org/10.1016/j.jmrt.2018.05.007.

[36] Silveira G M S. Estudo comparativo de juntas soldadas obtidas pelo processo GMAW convencional e GMAW com arame rotativo (GMAW-RE) [projeto final de graduação]. Rio de Janeiro: Centro Federal de Educação Tecnológica Celso Suckow da Fonseca; 2018.

[37] Guo N, Yanfei H, Zhang L, Zou Y. A modified heat source model for rotating arc welding. China Welding. 2011;20(3):58-63.

[38] Yadav PK, Abbas M, Patel S. Analysis of heat affected zone of mild steel specimen developed due to MIG welding. International Journal of Mechanical Engineering and Robtics Research. 2014;3(3):399-404.

[39] Zhang C, Li G, Gao M, Zeng XY. Microstructure and mechanical properties of narrow gap laser-arc hybrid welded $40 \mathrm{~mm}$ thick mild steel. Materials. 2017;10(2):1-14. http://dx.doi.org/10.3390/ma10020106. PMid:28772469.

[40] Gupta S, Datla SV, Sekhar C, Ali Shareef N. An experimental investigation on A36 carbon steel in submerged arc welded joints. International Journal of Mechanical Engineering and Technology. 2018;9(4):302-311.

[41] Flores IG, Arista BV, Dominguez JJG, Gonzalez CEC, Albarrán MAG, Villasana JP. Effect of torch weaving on the microstructure, tensile and impact resistances and fracture of the HAZ and weld bead by robotic GMAW process on ASTM A36 steel. Soldagem e Inspeção. 2017;22(1):72-86. http://dx.doi.org/10.1590/0104-9224/si2201.08.

[42] Corrêa FA. Propriedades mecânicas e microestruturais de juntas soldadas de aço carbono pelo processo híbrido laser GMAW [dissertação]. Rio de Janeiro: Centro Federal de Educação Tecnológica Celso Suckow da Fonseca; 2017.

[43] Jorge JCF, Souza LFG, Marouco ES, Santos OR Fo, Diniz JLC. Propriedades mecânicas e microestruturais de juntas soldadas pelo processo a arco submerso com elevado aporte térmico. Soldagem e Inspeção. 2015;20(3):347-358. http://dx.doi.org/10.1590/01049224/SI2004.04.

[44] Farneze HN, Jorge JCF, Souza LFG, Bott IS. Comparative study of high-strength steel weld metals obtained by the SMAW and FCAW processes for offshore applications and mooring chains. Welding International. 2010;24(12):903-910. http://dx.doi.org/10.1080/09507110903568943.

[45] Jorge JCF, Souza LFG, Couto JLS, Bott IS. Influence of chemical composition on the mechanical properties of high strength steel weld metals for application in mooring components. International Journal of Engineering and Technical Research. 2016;4(2):71-76.

[46] American Society for Testing and Materials. ASTM A-36-05: standard specification for carbon structural steel. West Conshohocken: ASTM; 2005. 4 p.

[47] Hasrizam WS, Muda W, Nasir NSM, Mamat S, Jamian S. Effect of welding heat input on microstructure and mechanical properties at coarse grain heat affected zone of ABS grade A steel. Journal of Engineering and Applied Sciences. 2015;10(20):9487-9495.

[48] Juan W, Yajiang L, Peng L. Effect of weld heat input on toughness and structure of HAZ of a new super-high strength steel. Bulletin of Materials Science. 2003;26(3):301-305. http://dx.doi.org/10.1007/BF02707450. 